\title{
Factors Associated with Attrition from HIV Care during the First Year after Antiretroviral Therapy Initiation in Kenya
}

Mia Liisa van der Kop ${ }^{1,2^{*}}$, Anna Mia Ekström ${ }^{1,3}$, Opondo Awiti-Ujiji1, Michael H Chung ${ }^{4}$, Daljeet Mahal ${ }^{5}$, Lehana Thabane ${ }^{6}$, Lawrence Gelmon ${ }^{7}$, Joshua Kimani ${ }^{8}$ and Richard Todd Lester ${ }^{2}$

${ }^{1}$ Department of Public Health Sciences, Karolinska Institute, Widerströmska Huset, Tomtebodavägen 18A, Stockholm 171-77, Sweden

${ }^{2}$ Division of Infectious Diseases, University of British Columbia, 828 West 10th Avenue, Vancouver, BC, V5Z 1M9, Canada

${ }^{3}$ Department of Infectious Diseases, I73, Karolinska University Hospital, 141 86, Stockholm, Sweden

${ }^{4}$ Departments of Global Health, Medicine and Epidemiology, University of Washington, 325 9th Avenue, Seattle, WA 98104, USA

${ }^{5}$ British Columbia Centre for Disease Control, 655 West 12th Avenue, Vancouver, BC, V5Z 4R4, Canada

${ }^{6}$ Department of Clinical Epidemiology and Biostatistics, McMaster University, 50 Charlton Avenue East, Hamilton, ON, L8N 4A6, Canada

${ }^{7}$ School Department of Medical Microbiology, University of Manitoba, 745 Bannatyne Avenue, Winnipeg, MB R3E OJ9, Canada

${ }^{8}$ Center for STD/HIV Research and Training, University of Nairobi, PO Box 19676-00202, Nairobi, Kenya

\begin{abstract}
Objectives: A secondary analysis of clinical trial data was used to: (1) estimate retention one year after antiretroviral therapy (ART) initiation; (2) determine factors associated with attrition; and (3) investigate whether participants deemed lost to follow-up (LTFU) were still engaged with the clinic through an interactive mobile phone intervention.

Methods: Between 2007 and 2008, adults initiating ART were recruited from three clinics in Kenya. Telephone and community tracing were undertaken to track participants who did not attend their 12-month appointment. Logistic regression was used to determine the association between an interactive text-messaging intervention, purposively selected clinical and socio-demographic factors, and attrition. Data on participant engagement with the text-messaging intervention were extracted from a communications log.

Results: Data from 538 participants were included. Retention in care at 6-months was $86.2 \%(461 / 535)$, decreasing to $81.8 \%(431 / 527)$ at 12 -months. At 12 -months, $53 \%(51 / 96)$ of attrition was due to death and $47 \%(45 / 96)$ to LTFU. A baseline CD4 count $<100$ was significantly associated with greater attrition (adjusted odds ratio $[A O R]=1.83,95 \% \mathrm{Cl}$ : 1.14-2.94). At 12-months, increased travel time to clinic was significantly associated with greater attrition in females (AOR 1.55, 95\% Cl: 1.12-2.14), but not in males (AOR 0.95, 95\% Cl: 0.65-1.39). Of 16 intervention arm participants considered LTFU at 6-months, 11 were in contact with the clinic through text-messaging. At 12-months, less than half (7/18) of participants considered LTFU who were enrolled in the service were participating in the intervention.

Conclusions: Our results confirm the association between a low baseline CD4 count and attrition, and suggest that travel time to the clinic is an important factor in retaining female patients. They highlight the importance of tracing studies to estimate retention and the opportunity provided by an interactive mobile health intervention to connect with patients considered LTFU.
\end{abstract}

Keywords: HIV/AIDS; Antiretroviral therapy; Sub-Saharan Africa; Retention; Attrition; Text messaging; Mobile phones

\section{Introduction}

\section{Background and rationale}

Retention in care is critical to maximizing patient outcomes and to the success of HIV care and treatment programmes. Retaining patients who are on antiretroviral therapy (ART) minimizes the likelihood of treatment interruptions and discontinuation, which not only reduces patient morbidity and mortality, [1] but also minimizes the risk of drug resistance [2] and viral transmission [3]. Despite these and other benefits, a recent systematic review indicates that only $80 \%$ of patients who initiate ART in sub-Saharan Africa remain in care at 12-months [4]. The review included several estimates of retention in care from HIV programmes in Kenya, many of which were based on studies that were not specifically intended to quantify retention in care [5]. Estimates from studies specially designed to evaluate patient retention were retrospective chart reviews [6,7]. These studies were limited in that they were unable to determine the true status of those considered lost to follow-up (LTFU), potentially underestimating patient retention in care. Reports indicate that when patients' activity is traced outside of the clinic at which patients initially registered, a significant proportion of patients are active in care elsewhere [8]. We investigated patient retention using data from a previously published randomized controlled trial (RCT) in Kenya involving adults initiating ART [9]. Participant tracing enabled us to ascertain the status of those who did not return to the clinic for their 12-month appointment, and helped distinguish between participants 'retained in clinic' versus those retained in care.

There is conflicting evidence on individual-level factors associated with retention in care, such as age, CD4 count, and travel time to clinic $[7,10,11]$. In addition to these factors, we investigated whether

*Corresponding author: Mia van der Kop, Division of Infectious Diseases, University of British Columbia, 828 West 10th Avenue, Vancouver, BC, V5Z IM9, Canada, Tel: +1 604875 4111; Fax: +1 604875 4013; E-mail: miavanderkop@gmail.com

Received June 25, 2014; Accepted September 20, 2014; Published September 30, 2014

Citation: van der Kop ML, Ekström AM, Awiti-Ujiji O, Chung MH, Mahal D, et al (2014) Factors Associated with Attrition from HIV Care during the First Year afte Antiretroviral Therapy Initiation in Kenya. J AIDS Clin Res 5: 354. doi:10.4172/2155 6113.1000354

Copyright: (C) 2014 van der Kop ML, et al. This is an open-access article distributed under the terms of the Creative Commons Attribution License, which permits unrestricted use, distribution, and reproduction in any medium, provided the original author and source are credited. 
an interactive text-messaging service was associated with improved retention in care. The text-message service, WelTel, was shown in an RCT to improve ART adherence and suppression of HIV-1 RNA load [9]. The intervention involved sending participants weekly check-in text messages. While published trial results presented the effect of the intervention on adherence and viral load, [9] they did not examine the effectiveness of the intervention to improve retention in care, or whether participants considered 'LTFU' were engaged with the clinic through the intervention. Overall, this cohort study provided a unique opportunity to investigate attrition from HIV care among those on treatment and the use of a mobile health intervention to improve engagement in care.

\section{Objectives}

1) Determine the proportion of individuals retained in HIV care at 6- and 12- months after initiating ART at three Kenyan clinics;

2) Identify socio-demographic and clinical factors associated with retention in HIV care;

3) Determine the proportion of participants considered lost to follow-up who were actively participating in the WelTel mobile health intervention, and the effect of the intervention on patient retention.

\section{Methods}

\section{Study design}

This cohort study used data collected from an RCT on the effectiveness of a text-messaging intervention to improve ART adherence. Adults initiating ART in Kenya were followed for up to 15 months to determine whether they were retained in care at six- and 12-months.

\section{Study setting and participants}

Between May 2007 and October 2008, the trial recruited participants from three clinics involved in ART provision scale-up: two in Nairobi and one government health centre in Kajiado, a large rural district. Patients were eligible for study participation if they were over 18 years old, initiating ART, had access to a mobile phone, and could communicate through text-messaging or have somebody text-message on their behalf. Follow-up study visits coincided with 6- and 12-month clinical visits. A community tracing study (not previously reported) was conducted in October 2009. Follow-up concluded in December 2009. Full details of the WelTel Kenyal trial and population have been published previously $[7,9]$.

\section{Variables}

\section{Outcomes}

Retention in care at 6- and 12-months: Participants were considered retained at 6-months if they attended their 6-month clinic appointment no later than three months (91 days) after their expected date of return with no subsequent visits during the study period. The proportion of participants who returned later than three months after their 6-month appointment is also reported. Twelve-month retention was defined as attendance at the clinic no later than three months (91 days) after the expected date of their 12-month visit. Retention in care was calculated as follows:

$$
\begin{aligned}
& \% \mathrm{RT}_{\mathrm{t}}=\left(\mathrm{I}_{0}-\mathrm{W}_{\mathrm{t}}-\mathrm{T}_{\mathrm{t}}-\mathrm{D}_{\mathrm{t}}-\mathrm{LTFU}_{\mathrm{t}}\right) /\left(\mathrm{I}_{0}-\mathrm{W}_{\mathrm{t}}-\mathrm{T}_{\mathrm{t}}\right) \\
& \mathrm{RT}_{\mathrm{t}}=\text { retained at time } t \\
& \mathrm{I}_{0}=\text { participants initiating ART }
\end{aligned}
$$

$$
\begin{aligned}
& \mathrm{W}_{t}=\text { withdrawals from the study by time } \mathrm{t} \\
& \mathrm{T}_{\mathrm{t}}=\text { transfers whose care status was unknown at time } \mathrm{t} \\
& \mathrm{D}_{\mathrm{t}}=\text { deaths by time } \mathrm{t} \\
& \mathrm{LTFU}_{\mathrm{t}}=\text { patients LTFU by time } t .
\end{aligned}
$$

Evidence of clinic visits included recorded visits, CD4 or viral load test dates, and ART pick-up dates. Transferred participants known to be active in care at their new facility were considered retained. Attrition is defined as 1-retention.

Data on LTFU and mortality were collected throughout the study period. All participants who missed their 12-month appointment were included in a telephone tracing study to ascertain their status. The study nurse called their primary telephone number and if required, their alternate numbers several times. Participants who could not be contacted were included in a community tracing study in which a research coordinator, driver and nurse attempted to locate the participant at their home. To protect participant confidentiality, the nature of the study team's visit was not disclosed to anyone other than the research participant. In the case of transfers, the clinic to which participants had transferred care was contacted to ascertain whether the participant was active in care. Participants LTFU included those who did not return to the clinic and were not contactable or traceable, or were confirmed as defaulting on care. Death was ascertained from clinical records and tracing studies.

Determinants of attrition and potential effect modifiers: Variables were purposively selected based on a systematic review of retention in care and individual studies examining retention in care in Kenya. Individual-level factors included: age (years) $(<30,30-39$, 40-49, $\geq 50$ ); [4] sex (male or female); [4] baseline CD4 count (cells/ $\left.\mathrm{mm}^{3}\right)(<100$ or $\geq 100)$; [4] disclosure of HIV status at baseline (yes or no); [11] and travel time (minutes) $(<30,31-60,61-120,>120)$ [11]. We investigated interaction between travel time and sex based on a priori information from a separate Kenyan study [11].

\section{The WelTel intervention}

For 12 months, every Monday morning, a nurse sent the text message "Mambo?" (KiSwahili for"How are you?") to patients in the intervention group to inquire about their status (Figure 1). Patients were

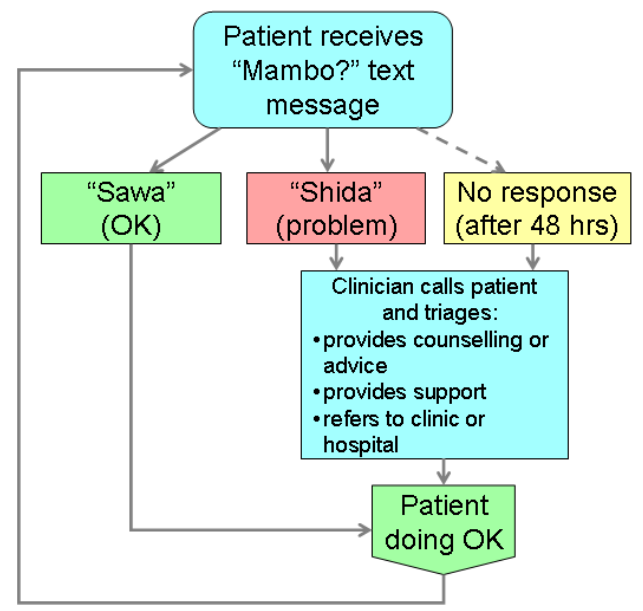

Figure 1: The WelTel intervention. 
instructed to respond within 48 hours either that they were doing well ("Sawa") or had a problem ("Shida"). The clinician called and provided triage to patients who indicated a problem or failed to respond.

Among participants in the intervention arm considered LTFU, engagement in the text-messaging service was determined by whether they were responding to the messages around the expected date of their 6- or 12-month visit. This timeframe included the month of their expected visit and up to three months after. The participants' level of responsiveness was categorized as non-responsive (responding to $0 \%$ of the messages); least responsive (responding to $1 \%-19 \%$ of the messages); poorly responsive (20\%-49\%); moderately responsive $(50 \%$ $79 \%)$; or highly responsive ( $\geq 80 \%)[12]$.

\section{Data sources and measurement}

Data on patients' demographic characteristics were collected using interviewer-administered questionnaires at the baseline visit. Followup questionnaires were administered and contact information was updated at 6- and 12-months. Study databases were cross-checked with clinical records to check the consistency of the data and to capture clinical data. Data on participation in the text-messaging service was extracted from the communications log, which documented all textmessage responses, instances of non-response, and mobile phone communications between healthcare providers and patients. Data were entered in Microsoft Access on a weekly basis. To minimize information bias, data on retention outcomes and other variables were collected and extracted in the same way for participants in both the control and intervention groups. To further minimize bias, the communications $\log$ was not used to ascertain study outcomes such as death, since this data source was specific to intervention arm participants, and may have resulted in more complete outcome information for participants who had received the text-messaging service.

\section{Study size}

A conservative rule is that logistic models should be used with 10 outcome events per predictor variable [13]. The total sample size, determined by the sample size required for the trial, [12] was 538 . There were 74 and 96 attrition events in this study at 6 - and 12-months respectively, which was adequate to build models with the five factors we selected.

\section{Ethics}

The original study protocol was approved by the University of Manitoba and Kenyatta National Hospital ethics review boards.

\section{Statistical analyses}

Descriptive analyses of the study population, including the proportions of patients retained in care at 6- and 12-months, were conducted in SPSS v14. For continuous variables (age and travel time), the linear assumption was checked using two methods: lowess smooth plots and quartile design variables [14]. Analyses were restricted to individuals with complete data.

To account for the clustered nature of the data (by study site), random effects logistic regression was used. First, univariable analyses were performed to assess the strength of the association between each factor and the outcome. Variables were then included in an initial multivariable model if they had a univariable $p$-value of $\leq 0.25$ or were considered important based on prior evidence (i.e. age). In the final models, variables were selected based on a significance threshold of $\mathrm{p}<0.05$. Nested models and likelihood ratio tests (LRT) were used to assess a variable's contribution to the model's fit and to examine interaction between sex and travel time. If interaction was significant $(\mathrm{p}<0.05)$, stratified results were reported. Nested models and LRTs were also used to determine whether to include a linear effect or indicator variables for ordered categorical variables. The goodness of model fit was checked by examining the accuracy of the quadrature approximation. A threshold of $10^{-4}$ in the relative difference in coefficients was used to determine whether the estimates were stable. Results are presented as estimated odds ratios (OR) with corresponding 95\% confidence intervals $(\mathrm{CI})$ and p-values. All p-values are two-sided and reported to three decimal places with those less than 0.001 reported as $\mathrm{p}<0.001$. Analyses were performed using Stata version 12 (Statacorp, College Station, TX).

\section{Results}

\section{Study population}

Data from all 538 participants who participated in the trial were included in the cohort study (Figure 2). The majority of participants were female $(65 \%)$ and had disclosed their HIV status at baseline (85\%) (Table 1). The median age was 36 years (first quartile [Q1]-third quartile [Q3]: 31-41), and the median baseline CD4 count was 149 cells/mm $\mathrm{mm}^{3}$ Q1-Q3: 82-218).

\section{Six-month retention in care}

The proportion of patients retained in care at six months was $86.2 \%$ (461/535), including eight participants who did not attend their 6-month appointment within the specified timeframe but attended a visit later in the study period. This calculation excluded three participants: two who had withdrawn from the study and one transfer whose care status was unknown. A second participant who had transferred was classified

\begin{tabular}{|c|c|c|c|c|c|c|}
\hline Variable & Total & $\begin{array}{l}\text { Retained in } \\
\text { care }\end{array}$ & $\begin{array}{l}\text { 6-month } \\
\text { attrition }\end{array}$ & Total & $\begin{array}{l}\text { Retained in } \\
\text { care }\end{array}$ & $\begin{array}{l}\text { 12-month } \\
\text { attrition }\end{array}$ \\
\hline \multicolumn{7}{|l|}{ Sex } \\
\hline Female & 349 & $312(89.4)$ & $37(10.6)$ & 345 & $292(84.6)$ & $53(15.4)$ \\
\hline Male & 186 & $149(80.1)$ & $37(19.9)$ & 182 & $139(76.4)$ & $43(23.6)$ \\
\hline \multicolumn{7}{|l|}{ Age (years) } \\
\hline$<30$ & 99 & $85(85.9)$ & $14(14.1)$ & 97 & 79 (81.4) & $18(18.6)$ \\
\hline 30-39 & 273 & $235(86.1)$ & $38(13.9)$ & 269 & $221(82.2)$ & $48(17.8)$ \\
\hline $40-49$ & 120 & $106(88.3)$ & $14(11.7)$ & 118 & $99(83.9)$ & $19(16.1)$ \\
\hline$\geq 50$ & 43 & $35(81.4)$ & $8(18.6)$ & 43 & $32(74.4)$ & $11(25.6)$ \\
\hline \multicolumn{7}{|c|}{ Baseline CD4 count (cells/mm³) } \\
\hline$<100$ & 163 & $123(75.5)$ & $40(24.5)$ & 159 & $118(74.2)$ & $41(25.8)$ \\
\hline$\geq 100$ & 367 & $334(91.0)$ & $33(9.0)$ & 363 & $309(85.1)$ & $54(14.9)$ \\
\hline \multicolumn{7}{|l|}{ Intervention } \\
\hline control & 263 & $222(84.4)$ & $41(15.6)$ & 262 & $209(79.8)$ & $53(20.2)$ \\
\hline intervention & 272 & $239(87.9)$ & $33(12.1)$ & 265 & $222(83.8)$ & $43(16.2)$ \\
\hline \multicolumn{7}{|c|}{ Travel time to clinic } \\
\hline$\leq 30$ minutes & 190 & $166(87.4)$ & $24(12.6)$ & 186 & $157(84.4)$ & $29(15.6)$ \\
\hline $\begin{array}{l}31-60 \\
\text { minutes }\end{array}$ & 179 & $156(87.2)$ & $23(12.8)$ & 176 & $147(83.5)$ & $29(16.5)$ \\
\hline $\begin{array}{l}61-120 \\
\text { minutes }\end{array}$ & 129 & $108(83.7)$ & $21(16.3)$ & 128 & $101(78.9)$ & $27(21.1)$ \\
\hline$>120$ minutes & 36 & $30(83.3)$ & $6(16.7)$ & 36 & $25(69.4)$ & $11(30.6)$ \\
\hline \multicolumn{7}{|c|}{ HIV disclosure at baseline } \\
\hline Disclosed & 455 & $393(86.4)$ & $62(13.6)$ & 447 & $369(82.6)$ & $78(17.4)$ \\
\hline $\begin{array}{l}\text { Not } \\
\text { disclosed }\end{array}$ & 80 & $68(85.0)$ & $12(15.0)$ & 80 & $62(77.5)$ & $18(22.5)$ \\
\hline
\end{tabular}

Table 1: Retention of participants at 6 and 12 months after ART initiation, by sociodemographic and clinical characteristics. 


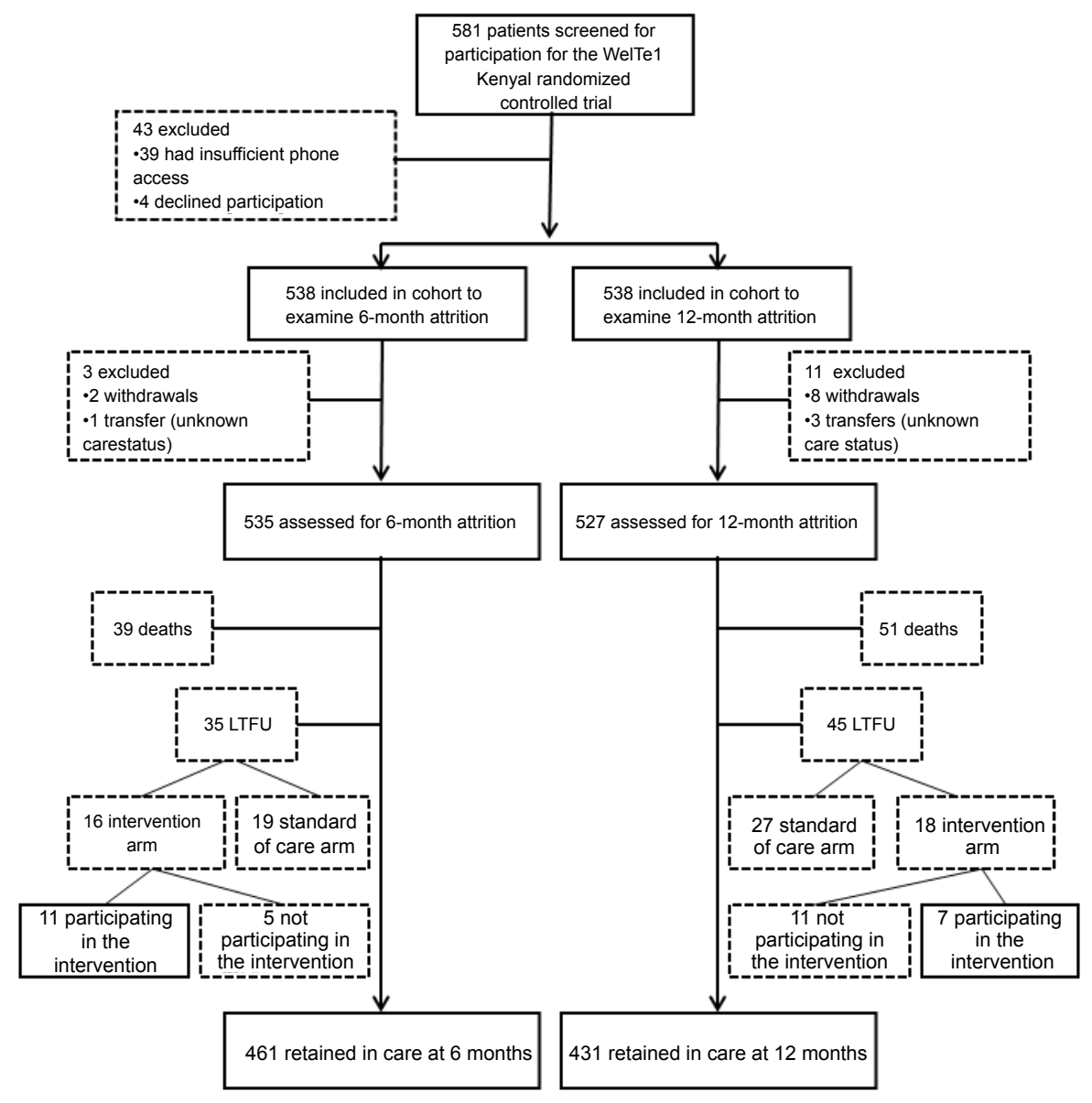

Figure 2: Flow of participants.

\begin{tabular}{|c|c|c|c|c|c|c|}
\hline \multirow[b]{2}{*}{ Variable } & \multicolumn{3}{|c|}{ Unadjusted (univariable) ORs } & \multicolumn{3}{|c|}{ Adjusted (multivariable) ORs } \\
\hline & OR & $95 \% \mathrm{Cl}$ & $\mathrm{p}$-value & OR & $95 \% \mathrm{Cl}$ & $p$-value \\
\hline Male gender & 2.00 & $1.21-3.30$ & 0.007 & 1.97 & $1.18-3.29$ & 0.010 \\
\hline $\mathrm{Age}^{*}$ & 0.92 & $0.68-1.13$ & 0.595 & & & \\
\hline CD4 $<100$ & 3.08 & $1.84-5.15$ & $<0.0001$ & 3.00 & $1.79-5.02$ & $<0.0001$ \\
\hline Intervention & 0.72 & $0.43-1.18$ & 0.189 & & & \\
\hline Travel time $^{\dagger}$ & 1.05 & $0.80-1.37$ & 0.735 & & & \\
\hline Non-disclosure of HIV status & 1.04 & $0.52-2.09$ & 0.903 & & & \\
\hline
\end{tabular}

${ }^{*} \mathrm{OR}$ corresponds to a decrease in the odds ratio per unit increase in age category ( $<30$ years, 30-39 years, $40-49$ years, $\geq 50$ years)

tOR corresponds to an increase in the odds ratio per unit increase in travel time category ( $<30$ minutes, $31-60$ minutes, $61-120$ minutes, $>120$ minutes)

OR odds ratio; $\mathrm{Cl}$ confidence interval

Table 2: Univariable and multivariable analysis of variables associated with attrition at 6-months in the trial cohort.

as retained as they were confirmed active in care at their new facility. Approximately half of attrition at 6-months was attributable to death (52.7\%: 39/74) while the other half resulted from lost to follow-up (47.3\%: 35/74).

In both univariable and multivariable analyses, males had twice the odds of attrition from care at 6-months, with an adjusted OR (AOR) of 1.97 (95\% CI 1.18 to 3.29) (Table 2). A baseline CD4 count of $<100$ was also associated with attrition (AOR 3.00; 95\%CI 1.79-5.02). Age, travel time, and disclosure were not associated with retention in care at 6-months. Although the odds of attrition at 6-months were lower among those in the intervention arm (OR 0.72), this finding was not statistically significant (95\% CI 0.43-1.18).

\section{Twelve-month retention in care}

The proportion of patients retained in care at 12 -months was $81.8 \%$ (431/527). This includes 25 participants who had transferred but were confirmed active in care at their new clinic. Transferred patients who were confirmed inactive at their new site $(n=2)$ were not considered 
retained and transfers with an unknown status were excluded $(\mathrm{n}=3)$. Participants whose status was unconfirmed at the time of their 12-month visit and whose status could not be ascertained through telephone tracing were traced in the community $(\mathrm{n}=19)$ (Table 3). Overall, $53.1 \%$ $(n=51 / 96)$ of attrition in the first year after ART initiation was due to death and $46.9 \%(n=45 / 96)$ to LTFU.

\begin{tabular}{|l|c|}
\hline Final status & $\mathbf{n ~ ( \% )}$ \\
\hline Active in care & $10(52.6)$ \\
\hline Transferred and confirmed inactive in care & $2(10.5)$ \\
\hline Death & $2(10.5)$ \\
\hline Unable to trace & $5(26.3)$ \\
\hline Total & $19(100.0)$ \\
\hline
\end{tabular}

Table 3: Outcomes of the participants involved in the community tracing study, October 2009

Similar to 6-month attrition, males and participants with a baseline CD4 count $<100$ (AOR 1.32, 95\% CI 1.14 to 2.94) had a greater likelihood of attrition at 12-months (Tables 4 and 5). When travel time was included in the multivariable model, it had a significant interactive effect with sex. Among females, increased travel time was associated with attrition, with an AOR of 1.55 per unit increase in category of travel time (95\% CI 1.12-2.14), while in males, travel time was not associated with attrition (AOR $0.95,95 \%$ CI 0.65-1.39). No association was found between disclosure, age, or the intervention and 12-month retention in care.

\begin{tabular}{|l|c|c|c|}
\hline Variable & OR & $95 \% \mathrm{Cl}$ & p-value \\
\hline Male gender & 1.62 & $1.03-2.56$ & 0.039 \\
\hline Age $^{*}$ & 0.97 & $0.74-1.27$ & 0.815 \\
\hline CD4 <100 & 1.89 & $1.18-3.01$ & 0.008 \\
\hline Intervention & 0.74 & $0.47-1.17$ & 0.199 \\
\hline Travel time $^{\dagger}$ & 1.20 & $0.94-1.52$ & 0.148 \\
\hline Non-disclosure of HIV status & 1.25 & $0.69-2.27$ & 0.470 \\
\hline
\end{tabular}

${ }^{*} \mathrm{OR}$ corresponds to a decrease in the odds ratio per unit increase in age category ( $<30$ years, $30-39$ years, $40-49$ years, $\geq 50$ years)

tOR corresponds to an increase in the odds ratio per unit increase in travel time category (<30 minutes, 31-60 minutes, 61-120 minutes, $>120$ minutes) OR odds ratio; $\mathrm{Cl}$ confidence interval

Table 4: Univariable analysis of variables associated with attrition at 12-months in the trial cohort.

\begin{tabular}{|l|c|c|c|c|}
\hline \multicolumn{2}{|l|}{ Variable } & OR & $95 \% \mathrm{Cl}$ & p-value \\
\hline CD4 $<100$ & & 1.83 & $1.14-2.94$ & 0.012 \\
\hline Travel time $^{\dagger \S}$ & Females $^{\dagger}$ & 1.55 & $1.12-2.14$ & 0.008 \\
\hline & Males $^{\ddagger}$ & 0.95 & $0.65-1.39$ & 0.805 \\
\hline
\end{tabular}

t§OR corresponds to an increase in the odds ratio per unit increase in travel time category

₹OR corresponds to a decrease in the odds ratio per unit increase in travel time category

†Stratified results are presented because of significant interaction between sex and travel time (likelihood ratio test $p$-value $=0.030$ ). Stratified results are adjusted for CD4 count.

OR odds ratio; $\mathrm{Cl}$ confidence interval

Table 5: Final multivariable model of variables associated with attrition at 12-months in the trial cohort.

\section{Participation in the WelTel SMS service among those 'lost to follow-up'}

Of the 35 participants considered lost to follow-up at 6-months, 16 were in the intervention arm. Despite being technically classified as lost to follow-up at 6-months, the majority of these participants $(n=11 / 16)$ were still in contact with the clinic through the text-messaging service. Categorized by level of responsiveness to the intervention, 36\% $(n=4 / 11)$ were highly responsive to the intervention; $27 \%(n=3 / 11)$ were poorly responsive; and the remainder were least responsive $(36 \%: n=4 / 11)$.

At twelve-months, 18 of the 45 participants LTFU were in the intervention arm. Less than half (39\%: 7/18) of these participants were still participating in the intervention. Of the seven participants who were responding, six were highly responsive to the intervention and one was 'least responsive. There was a borderline significant decrease $(p=0.08)$ in the proportion of those engaged through the intervention at 6 -months compared to at 12 -months (69\% v. $39 \%)$.

\section{Discussion}

\section{Key results}

Among a cohort of individuals initiating ART at three clinics in Kenya, retention in care at 6 -months was $86 \%$, decreasing to $82 \%$ at one year. Patients who had transferred and were confirmed active in care at their new facility accounted for $6 \%$ of those considered retained at one year. Death was the most common cause of attrition at 12-months (53\%); $47 \%$ of attrition was due to LTFU. Males and individuals with a CD 4 count $<100$ at baseline were less likely to be retained in care at both time points. While travel time to clinic was not a factor in retaining males, females with a longer travel time were less likely to be retained at 12-months. Although the WelTel text-messaging intervention did not have a statistically significant effect on attrition, over half of those in the intervention arm considered LTFU at 6-months were still communicating with the clinic through the text-message service around the time of attrition. At one year, one-third of those defined as LTFU were still participating in the SMS intervention.

\section{Strengths and limitations of the study}

One of this study's principal strengths is that we conducted telephone and community tracing to ascertain participants' status at 12-months. Without participant tracing, it would have been assumed that those who did not come back to the clinic were LTFU. In many instances; these participants were actively engaged in care at another facility. As a result, our estimates of retention more closely reflect 'retention in care' than 'retention in clinic'. Additional study strengths include highly complete information on baseline variables such as CD4 count, disclosure and travel time, and enhanced external validity through the multi-site nature of the study.

The primary limitation of this study is that it is based on the secondary use of clinical trial data; therefore, it is subject to the inclusion and exclusion criteria designed for the purposes of the original trial. Accordingly, individuals with insufficient mobile phone access were excluded. Although this represents less than $10 \%$ of the population screened for trial participation, ideally, this group would have been included. Despite the fact that study visits coincided with regularly scheduled clinical visits, our results may have been affected by the Hawthorne effect, whereby participation in a clinical trial may have artificially inflated estimates of retention compared to a nontrial population. Another limitation is that despite efforts to trace all participants LTFU, we were unable to ascertain the outcomes of some participants. Finally, we relied on clinic records and patients' contacts to capture death data, a method that may be less reliable than vital statistics registration records had they been available.

\section{Comparability with other studies}

The proportion of individuals retained in care at 6-months in our 
cohort is the same as that found in an updated systematic review on retention in sub-Saharan Africa from a similar time period $(86.2 \%$ versus $86.1 \%$ respectively) [4]. However, our estimate of retention at 12 -months is greater than 12-month estimates in both the original and updated reviews of retention. In most cases, our estimate also stands out as markedly higher compared to estimates from other Kenyan studies $[6,7,10,15,16]$. Although the lack of consistency between our estimate and those in the review may be due to a variety of factors (e.g. differences in the type or size of the HIV care program, time period, populations generally, etc., or differences arising because we studied a clinical trial versus non-trial population), we believe an important factor is that in our cohort, patients who transferred and were known to be active in care at their new facility were considered retained. Conversely, patients who transferred care in most other studies were considered LTFU, potentially underestimating retention. These divergent results highlight the importance of tracing patients considered LTFU to reliably estimate retention. It also provides evidence to support the hypothesis that many patients considered LTFU may have informally transferred care to another facility, rather than dropped out of care completely [17].

Rosen and Fox's systematic review found that at a cohort-level, a median starting CD4 count $<100$ and age $<36$ were associated and with a greater odds of attrition [18]. Our individual-level study also found that a low baseline CD4 count was associated with greater attrition, but that age was not. The lack of association between age and attrition is consistent with two other Kenyan studies $[7,10]$ but not with OchiengOoko's more recent study, [11] whose findings more closely reflect those in the systematic review. The Ochieng-Ooko study's sample size was substantial $(n>20,000$ patients on ART), increasing their ability to detect a significant effect. They also limited their 'after cART initiation' analysis to patients who had attended at least one follow-up visit, resulting in different inclusion criteria and possibly giving rise to differences in the results. These same reasons may also explain why they detected increased attrition among men who had not disclosed their status at baseline, whereas we did not find such an association.

Importantly, this study found that increased travel time to the clinic is a factor in retaining women at one-year but not men. OchiengOoko found a similar effect, but only among pre-ART patients [11]. Differences in our findings among patients taking ART may be due to Ochieng-Ooko et al. differing inclusion criteria, and/or their dichotomization of travel time at one hour (versus using a multicategory or continuous variable), which may have resulted in a loss of information [19]. In our study, evidence of a causal effect of travel time on retention in care is strengthened by the strong linear trend between increasing travel time to clinic and attrition, with approximately a four-fold increase in the odds of attrition among females with greater than two hours travel time compared to those able to reach the clinic in under half an hour. Increasing decentralization of care may help ameliorate the problem of having to travel a long time to reach a clinic. In the meantime, in-depth research into the reasons behind why this barrier exists is needed. If it stems from the potentially greater costs of transportation associated with increased travel time, travel vouchers may be of benefit. If it is due to a lack of transportation, time costs, or to other reasons, alternative strategies are required. Mobile phones may provide a unique opportunity to facilitate communication between patients and healthcare providers, regardless of distance.

Although participants who received the WelTel intervention had greater odds of retention, this study was underpowered to achieve statistical significance for this outcome. A larger trial is currently underway to examine whether the WelTel intervention, through provision of ongoing support and regular communication, can improve retention in care among newly diagnosed individuals in Kenya [20]. Nevertheless, a key finding in this study was that many participants considered LTFU, particularly at 6-months, were still in communication with the clinic through the service. With the programmatic implementation of service at several sites in Kenya, the intervention may provide an opportunity to encourage patient reengagement in ART care.

\section{Conclusions}

Our results confirm the association between a low baseline CD4 count and attrition, and suggest that travel time to the clinic is an important factor in retaining female patients. They highlight the importance of tracing studies to estimate retention and the opportunity provided by an interactive mobile health intervention to connect with patients considered LTFU.

\section{Acknowledgements}

We thank the healthcare providers, research staff and patients who participated in the study. This manuscript would not have been possible without the help of Sarah Karanja, who was involved in conducting the participant tracing and provided us with the data. Sections of this manuscript were submitted as assignments for an Academic Writing Course through the Swedish Research School for Global Health. We are grateful to the course instructor, Karyn Sandström, for her suggestions and comments.

The original trial was funded by the US President's Emergency Plan for AIDS Relief (PEPFAR) through the CDC cooperative agreement 5U62PS024510 as a public-health evaluation (PHE:KE07.0045). MLVDK is supported by a Canadian Institutes of Health Research (CIHR) Doctoral Award - Doctoral Foreign Study Award (October 2012), offered in partnership with the CIHR Strategy for PatientOriented Research and the CIHR HIVIAIDS Research Initiative. RTL is supported by the Grand Challenges Canada Stars in Global Health program.

\section{References}

1. Palella FJ Jr, Delaney KM, Moorman AC, Loveless MO, Fuhrer J, et al. (1998) Declining morbidity and mortality among patients with advanced human immunodeficiency virus infection. HIV Outpatient Study Investigators. N Engl J Med 338: 853-860.

2. Sethi AK, Celentano DD, Gange SJ, Moore RD, Gallant JE (2003) Association between adherence to antiretroviral therapy and human immunodeficiency virus drug resistance. Clin Infect Dis 37: 1112-1118.

3. Hosseinipour M, Cohen MS, Vernazza PL, Kashuba AD (2002) Can antiretrovira therapy be used to prevent sexual transmission of human immunodeficiency virus type 1? Clin Infect Dis 34: 1391-1395.

4. Fox MP, Rosen S (2010) Patient retention in antiretroviral therapy programs up to three years on treatment in sub-Saharan Africa, 2007-2009: systematic review. Trop Med Int Health 15 Suppl 1: 1-15.

5. Carillo de Casas E, Cox H, Ferreyra P, Isaakidis C, Mansilla MJ, et al. (2006) Gender differences in a large-scale HAART program in western Kenya. Toronto, Canada.

6. Macharia DK, Chang LW, Lule G, Owili DM, Tesfaledet G, et al. (2003) Antiretroviral therapy in the private sector of Nairobi, Kenya: a review of the experience of five physicians. AIDS 17: 938-940.

7. Unge C, Södergård B, Ekström AM, Carter J, Waweru M, et al. (2009) Challenges for scaling up ART in a resource-limited setting: a retrospective study in Kibera, Kenya. J Acquir Immune Defic Syndr 50: 397-402.

8. Geng EH, Glidden DV, Bwana MB, Musinguzi N, Emenyonu N, et al. (2011) Retention in care and connection to care among HIV-infected patients on antiretroviral therapy in Africa: estimation via a sampling-based approach PLoS One 6: e21797.

9. Lester RT, Ritvo P, Mills EJ, Kariri A, Karanja S, et al. (2010) Effects of a mobile phone short message service on antiretroviral treatment adherence in Kenya (WelTel Kenya1): a randomised trial. Lancet 376: 1838-1845.

10. Karcher H, Omondi A, Odera J, Kunz A, Harms G (2007) Risk factors for treatment denial and loss to follow-up in an antiretroviral treatment cohort in Kenya. Trop Med Int Health 12: 687-694. 
Citation: van der Kop ML, Ekström AM, Awiti-Ujiji O, Chung MH, Mahal D, et al. (2014) Factors Associated with Attrition from HIV Care during the First Year after Antiretroviral Therapy Initiation in Kenya. J AIDS Clin Res 5: 354. doi:10.4172/2155-6113.1000354

11. Ochieng-Ooko V, Ochieng D, Sidle JE, Holdsworth M, Wools-Kaloustian K, et al. (2010) Influence of gender on loss to follow-up in a large HIV treatment programme in western Kenya. Bull World Health Organ 88: 681-688.

12. Lester RT, Mills EJ, Kariri A, Ritvo P, Chung M, et al. (2009) The HAART cell phone adherence trial (WelTel Kenya1): a randomized controlled trial protocol. Trials 10: 87 .

13. Peduzzi P, Concato J, Kemper E, Holford TR, Feinstein AR (1996) A simulation study of the number of events per variable in logistic regression analysis. J Clin Epidemiol 49: 1373-1379.

14. Hosmer DWJ, Lemeshow S, Studivant RX (2013) Applied Logistic Regression. Hoboken, NJ: John Wiley \& Sons, Inc.

15. Wools-Kaloustian K, Kimaiyo S, Diero L, Siika A, Sidle J, et al. (2006) Viability and effectiveness of large-scale HIV treatment initiatives in sub-Saharan Africa: experience from western Kenya. AIDS 20: 41-48.
16. Hawkins C, Achenbach C, Fryda W, Ngare D, Murphy R (2007) Antiretroviral durability and tolerability in HIV-infected adults living in urban Kenya. J Acquir Immune Defic Syndr 45: 304-310.

17. Marson KG, Tapia K, Kohler P, McGrath CJ, John-Stewart GC, et al. (2013) Male, mobile, and moneyed: loss to follow-up vs. transfer of care in an urban African antiretroviral treatment clinic. PLoS One 8: e78900.

18. Rosen S, Fox MP, Gill CJ (2007) Patient retention in antiretroviral therapy programs in sub-Saharan Africa: a systematic review. PLoS Med 4: e298.

19. Royston P, Altman DG, Sauerbrei W (2006) Dichotomizing continuous predictors in multiple regression: a bad idea. Stat Med 25: 127-141.

20. van der Kop ML, Ojakaa DI, Patel A, Thabane L, Kinagwi K, Ekström AM, et al. (2013) The effect of weekly short message service communication on patient retention in care in the first year after HIV diagnosis: study protocol for a randomised controlled trial (WelTel Retain). BMJ Open 3: e003155. 